RIMS-1690

Computation of the Shapley Value of Minimum Cost Spanning Tree Games: \#P-Hardness and Polynomial Cases

By

Kazutoshi ANDO

February 2010

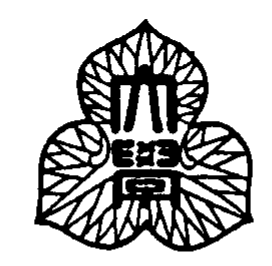

京都大学 数理解析研究所

RESEARCH INSTITUTE FOR MATHEMATICAL SCIENCES

KYOTO UNIVERSITY, Kyoto, Japan 


\title{
Computation of the Shapley Value of Minimum Cost Spanning Tree Games: \#P-Hardness and Polynomial Cases
}

\author{
Kazutoshi Ando*
}

February 2010

\begin{abstract}
We show that computing the Shapley value of minimum cost spanning tree games is \#P-hard even if the cost functions are restricted to be $\{0,1\}$-valued. The proof is by a reduction from counting the number of minimum 2-terminal vertex cuts of an undirected graph, which is \#P-complete. We also investigate minimum cost spanning tree games whose Shapley values can be computed in polynomial time. We show that if the cost function of the given network is a subtree distance, which is a generalization of a tree metric, then the Shapley value of the associated minimum cost spanning tree game can be computed in $\mathrm{O}\left(n^{4}\right)$ time, where $n$ is the number of players.
\end{abstract}

\section{Introduction}

This paper deals with the computational complexity of the Shapley value [22] of minimum cost spanning tree games. Suppose that $\mathrm{K}_{V}$ is the complete graph with vertex set $V$ and a function $c$ which assigns a nonnegative cost $c(v, w)$ to each edge $\{v, w\}$ of $\mathrm{K}_{V}$ is given. A minimum cost spanning tree game (MCST game for short) is a cooperative (cost) game $(N, \tilde{c})$ defined as follows. The set of players is $N=V-\{r\}$, where $r \in V$ is a designated vertex, and for each $X \subseteq V \tilde{c}(X)$ is the cost of a minimum cost spanning tree of the subgraph of $\mathrm{K}_{V}$ induced by $X \cup\{r\}$. Minimum cost spanning tree games are introduced in the seminal paper [6] by Bird and fundamental theory was developed in [6], [13], [14] and [15].

There is a considerably rich literature on MCST games by economists, studying mostly axiomatic properties of several solution concepts for them. See, e.g., [8], [19], [17], [11] and [7]. In contrast, there is only few literature on the computational complexity of MCST games. (Faigle, Kern and Kuipers [10] show that computing the nucleolus of the MCST games is NP-hard and Faigle, Kern, Fekete and Hochstättler [9]

${ }^{*}$ Research Institute for Mathematical Sciences, Kyoto University, Kyoto 606-8502, Japan. On leave from Faculty of Engineering, Shizuoka University, Hamamatsu 432-8561, Japan. Email: ando@sys.eng.shizuoka.ac.jp 
show that testing membership in the core of MCST games is co-NP-complete.) Especially, the computational complexity of the Shapley value of the MCST games has been an open problem.

In this paper, we show that computing the Shapley value of MCST games is \#P-hard even if the cost functions are restricted to be $\{0,1\}$-valued, where we use a reduction from counting the number of minimum 2-terminal vertex cuts of an undirected graph. We also investigate MCST games whose Shapley values can be computed in polynomial time. We show that if the cost function of the given network is a subtree distance [16], which is a weaker notion of tree metric (see [21]), then the Shapley value of the associated game can be computed in $\mathrm{O}\left(n^{4}\right)$ time, where $n$ is the number of players. This class of MCST games properly includes the formerly known subclass of MCST games for which there exists a polynomial time algorithm computing the Shapley value (see [5], [2]).

The rest of this paper is organized as follows. In Section 2, we give basic definitions and review fundamental results on MCST games. In Section 3, we prove the \#Phardness of the Shapley value of MCST games. In Section 4, we consider cases where the Shapley value can be computed in polynomial time. Section 5 gives summary and concluding remarks of this paper.

\section{Basic Definitions and Preliminaries}

We denote by $\mathbb{R}$ the set of real numbers and by $\mathbb{R}_{+}$the set of nonnegative real numbers. For a subset $X$ and a single element $y$, we write $X \cup y$ instead of $X \cup\{y\}$. The set difference of two sets $X$ and $Y$ is denoted by $X-Y$ and we write $X-y$ instead of $X-\{y\}$ if $Y=\{y\}$ is a singleton.

\subsection{Cooperative games and the Shapley value}

A cooperative (cost) game $(N, f)$ is a pair of a finite set $N$ and a function $f: 2^{N} \rightarrow \mathbb{R}$ with $f(\emptyset)=0$. We call $N$ the set of the players and the function $f$ is called the characteristic function. In the sequel, we sometimes call a cooperative game simply a game.

The Shapley value $\Phi(f) \in \mathbb{R}^{N}$ of game $(N, f)$ is defined as

$$
\Phi_{v}(f)=\sum_{v \notin X \subseteq N} \frac{|X| !(n-|X|-1) !}{n !}(f(X \cup v)-f(X)) \quad(v \in N) .
$$

See a survey [23] for axiomatic characterizations of the Shapley value. For our purpose, an important feature of the Shapley value is the linearity: for any two games $(N, f),(N, g)$ and scalers $\lambda, \mu \in \mathbb{R}$, we have

$$
\Phi(\lambda f+\mu g)=\lambda \Phi(f)+\mu \Phi(g)
$$

where game $(N, \lambda f+\mu g)$ is defined by $(\lambda f+\mu g)(X)=\lambda f(X)+\mu g(X)(X \subseteq N)$.

For game $(N, f)$ and $U \subseteq N$, the restriction of $(N, f)$ to $U$ is a game $(U, f \mid U)$ defined by $(f \mid U)(X)=f(X)(X \subseteq U)$. 
Lemma 2.1: Let $(N, f)$ be a cooperative game and $U \subseteq N$. For a game $(N, f * U)$ defined by $(f * U)(X)=f(X \cap U)(X \subseteq N)$, we have

$$
\Phi_{v}(f * U)=\left\{\begin{array}{ll}
\Phi_{v}(f \mid U) & \text { if } v \in U, \\
0 & \text { otherwise }
\end{array} \quad(v \in N) .\right.
$$

(Proof) If $v \notin U$, then we have $(f * U)(X \cup v)=(f * U)(X)$ for all $X \subseteq N$. Hence, $\Phi_{v}(f * U)=0$. Suppose that $v \in U$. Then, we have

$$
\begin{aligned}
\Phi_{v}(f * U) & =\sum_{X \subseteq N-v} \frac{|X| !(n-|X|-1) !}{n !}(f((X \cap U) \cup v)-f(X \cap U)) \\
& =\sum_{Y \subseteq U-v} \sum_{Z \subseteq N-U} \frac{|Y \cup Z| !(n-|Y \cup Z|-1) !}{n !}(f(Y \cup v)-f(Y)) \\
& =\sum_{Y \subseteq U-v} \sum_{z=0}^{n-u}\left(\begin{array}{c}
n-u \\
z
\end{array}\right) \frac{(y+z) !(n-y-z-1) !}{n !}(f(Y \cup v)-f(Y)),
\end{aligned}
$$

where we set $u=|U|, y=|Y|$ and $z=|Z|$. Since

$$
\begin{aligned}
& \sum_{z=0}^{n-u} \frac{(n-u) !}{z !(n-u-z) !} \frac{(y+z) !(n-y-z-1) !}{n !} \\
& =\frac{(n-u) ! y !(u-y-1) !}{n !} \sum_{z=0}^{n-u} \frac{(y+z) !}{y ! z !} \frac{(n-y-z-1) !}{(n-u-z) !(u-1) !} \\
& =\frac{(n-u) ! y !(u-y-1) !}{n !} \sum_{z=0}^{n-u}\left(\begin{array}{c}
y+z \\
z
\end{array}\right)\left(\begin{array}{c}
n-y-z-1 \\
n-u-z
\end{array}\right) \\
& =\frac{(n-u) ! y !(u-y-1) !}{n !}\left(\begin{array}{c}
n \\
n-u
\end{array}\right) \\
& =\frac{y !(u-y-1) !}{u !}
\end{aligned}
$$

we have

$$
\Phi_{v}(f * U)=\sum_{Y \subseteq U-v} \frac{y !(u-y-1) !}{u !}(f(Y \cup v)-f(Y))=\Phi_{v}(f \mid U) .
$$

\subsection{The Shapley value of MCST Games}

All graphs we consider in this paper are simple undirected graphs (without self-loop and parallel edges). For a graph $G=(V, E)$ and $U \subseteq V$, we denote by $G[U]$ the subgraph of $G$ induced by $U$. We denote by $\mathrm{K}_{V}$ the complete graph with the vertex set being $V$, i.e., $\mathrm{K}_{V}=\left(V,\left(\begin{array}{c}V \\ 2\end{array}\right)\right)$, where $\left(\begin{array}{c}V \\ 2\end{array}\right)=\{\{v, w\} \mid v, w \in V, v \neq w\}$. For a graph $G=(V, E)$ a subgraph $H=(W, F)$ is called a spanning tree if $V=W$ and $H$ is a 
tree. We also say that $F$ is a spanning tree of $G=(V, E)$ if $H=(W, F)$ is a spanning tree of $G$.

Let $\mathrm{K}_{V}=\left(V,\left(\begin{array}{l}V \\ 2\end{array}\right)\right)$ be the complete graph with vertex set $V$ and let $c:\left(\begin{array}{l}V \\ 2\end{array}\right) \rightarrow \mathbb{R}_{+}$ be a function. We call such a pair $\left(\mathrm{K}_{V}=\left(V,\left(\begin{array}{c}V \\ 2\end{array}\right)\right), c\right)$ a network. For each subset $T \subseteq\left(\begin{array}{l}V \\ 2\end{array}\right)$, we define the $\operatorname{cost} c(T)$ of $T$ by $c(T)=\sum_{\{v, w\} \in T} c(v, w)$.

Let $\left(\mathrm{K}_{V}, c\right)$ be a network with a designated vertex $r$ called the source. The minimum cost spanning tree game (or MCST game for short) associated with network $\left(\mathrm{K}_{V}, c\right)$ is a cooperative game $(N, \tilde{c})$ defined as follows. The set of players is $N=V-r$ and $\tilde{c}: 2^{N} \rightarrow \mathbb{R}$ is defined by

$$
\tilde{c}(X)=\min \left\{c(T) \mid T \subseteq\left(\begin{array}{c}
X \cup r \\
2
\end{array}\right) \text { is a spanning tree of } \mathrm{K}_{X \cup r}\right\} \quad(X \subseteq N),
$$

where $\mathrm{K}_{X \cup r}$ is the complete subgraph of $\mathrm{K}_{V}$ induced by $X \cup r$.

For a network $\left(\mathrm{K}_{V}=\left(V,\left(\begin{array}{c}V \\ 2\end{array}\right)\right), c\right)$, let the distinct values of positive $c(v, w)$ 's be

$$
(0<) \gamma_{1}<\cdots<\gamma_{p}
$$

and let $\gamma_{0}=0$. For each $i=1, \cdots, p$ define $c_{i}:\left(\begin{array}{c}V \\ 2\end{array}\right) \rightarrow\{0,1\}$ by

$$
c_{i}(v, w)= \begin{cases}1 & \text { if } \gamma_{i} \leq c(v, w), \quad\left(\{v, w\} \in\left(\begin{array}{l}
V \\
2
\end{array}\right)\right) . \\
0 & \text { otherwise }\end{cases}
$$

We have

$$
c=\sum_{i=1}^{p}\left(\gamma_{i}-\gamma_{i-1}\right) c_{i} .
$$

Furthermore, we have the following proposition due to Norde, Moretti and Tijs [19]. The proof is essentially the same as that of [19] but is slightly shorter.

Proposition 2.2 (Norde, Moretti and Tijs [19]): Let $\left(\mathrm{K}_{V}, c\right)$ be a network with source $r$. Then, $\tilde{c}: 2^{N} \rightarrow \mathbb{R}$ is decomposed as

$$
\tilde{c}=\sum_{i=1}^{p}\left(\gamma_{i}-\gamma_{i-1}\right) \widetilde{c_{i}},
$$

where $c_{i}:\left(\begin{array}{c}V \\ 2\end{array}\right) \rightarrow\{0,1\}$ is defined by (2.16) for $i=1, \cdots, p$.

(Proof) We proceed by induction on $p \geq 0$. For $p=0,1$ we have nothing to prove. Suppose that $k>2$ and the assertion of the present proposition is true for $p=k-1$.

Let us consider $c^{\prime}:\left(\begin{array}{c}V \\ 2\end{array}\right) \rightarrow \mathbb{R}_{+}$defined by

$$
c^{\prime}(v, w)=\left\{\begin{array}{ll}
c(v, w)-\gamma_{1} & \text { if } c(v, w)>0, \\
0 & \text { otherwise }
\end{array}\left(\{v, w\} \in\left(\begin{array}{l}
V \\
2
\end{array}\right)\right) .\right.
$$

Then, we have

$$
c^{\prime}=c-\gamma_{1} c_{1}=\sum_{i=2}^{p}\left(\gamma_{i}-\gamma_{i-1}\right) c_{i} .
$$

Let $X \subseteq N$ and let $T \subseteq\left(\begin{array}{c}X \cup r \\ 2\end{array}\right)$ be a minimum cost spanning tree of $\left(\mathrm{K}_{X \cup r}, c_{1}\right)$. Define $T(0)=\left\{\{v, w\} \mid\{v, w\} \in T, c_{1}(v, w)=0\right\}$. Since for each $\{v, w\} \in T(0)$ we 
have $c^{\prime}(v, w)=0$, it follows from the validity of the greedy algorithm of Kruskal [18] that there exists a minimum cost spanning tree $T^{\prime}$ of $\left(\mathrm{K}_{X \cup r}, c^{\prime}\right)$ such that $T(0) \subseteq T^{\prime}$. Then, we have $c_{1}\left(T^{\prime}\right) \leq|X|-|T(0)|=c_{1}(T)$, and hence, $T^{\prime}$ is also a minimum cost spanning tree of $\left(\mathrm{K}_{X \cup r}, c_{1}\right)$. Therefore, we have

$$
\tilde{c}(X)=\gamma_{1} \widetilde{c_{1}}(X)+\widetilde{c^{\prime}}(X) .
$$

It follows from the induction hypothesis that

$$
\tilde{c}(X)=\sum_{i=1}^{p}\left(\gamma_{i}-\gamma_{i-1}\right) \widetilde{c}_{i}(X) .
$$

This completes the proof of the present lemma.

We have from Proposition 2.2 and the linearity of the Shapley value the following proposition.

Proposition 2.3: Suppose that $\left(\mathrm{K}_{V}, c\right)$ be a network and $c$ is decomposed as in (2.17). Then, we have

$$
\Phi(\tilde{c})=\sum_{i=1}^{p}\left(\gamma_{i}-\gamma_{i-1}\right) \Phi\left(\widetilde{c}_{i}\right) .
$$

Therefore, computation of the Shapley value of an MCST game is reduced to that of an MCST game with a $\{0,1\}$-valued cost function.

Suppose that $\left(\mathrm{K}_{V}, c\right)$ is a network with source $r \in V$, where $c$ is $\{0,1\}$-valued. Let us consider the graph $G(0)=(V, E(0))$, where

$$
E(0)=\left\{\{v, w\} \mid\{v, w\} \in\left(\begin{array}{c}
V \\
2
\end{array}\right), c(v, w)=0\right\} .
$$

It is straightforward to see the following propositions.

Proposition 2.4: Suppose that $\left(\mathrm{K}_{V}, c\right)$ is a network with source $r \in V$, where $c$ is $\{0,1\}$-valued and graph $G(0)=(V, E(0))$ is defined by (2.24). Then, $\tilde{c}(X)$ is equal to the number of connected components of $G(0)[X \cup r]-1$ for each $X \subseteq N$.

Note that if $r$ is an isolated vertex of $G(0)[X \cup r]$, then $\tilde{c}(X)$ is the number of connected components of $G(0)[X]$.

Proposition 2.5: Suppose that $\left(\mathrm{K}_{V}, c\right)$ is a network with source $r \in V$, where $c$ is $\{0,1\}$-valued and graph $G(0)=(V, E(0))$ is defined by (2.24). For a clique $Q \subseteq V$ of $G(0)$, we have

(i) If $r \notin Q$ and $r$ is an isolated vertex of $G(0)$, then $\Phi_{v}(\tilde{c} \mid Q)=\frac{1}{|Q|}$ for all $v \in Q$.

(ii) If $r \in Q$, then $\Phi_{v}(\tilde{c} \mid Q-r)=0$ for all $v \in Q-r$. 


\section{3. \#P-Hardness}

In this section, we show that the following problem is \#P-hardness even if the cost functions $c$ are restricted to be $\{0,1\}$-valued.

Definition 3.1: MCSTG-SHAPLEY: Given a network $\left(\mathrm{K}_{V}, c\right)$ with source $r$, we are asked to compute the Shapley value of game $(N, \tilde{c})$, where $\tilde{c}: 2^{N} \rightarrow \mathbb{R}$ is defined by (2.14).

The proof is by a reduction from counting the number of minimum 2-terminal vertex cuts of an undirected graph.

Let $G=(N, E)$ be an undirected graph with two terminal vertices $s, t \in N$ $(s \neq t)$. Vertex set $X \subseteq N-\{s, t\}$ is called an $s$-t vertex cut if $s$ and $t$ are not in the same connected component of $G-X$, where $G-X$ is the subgraph of $G$ induced by $N-X$.

Definition 3.2: \#MINIMUM s-t VERTEX CUT: Given an undirected graph $G=$ $(N, E)$ and distinct vertices $s, t \in N$, we are asked to compute the number of minimum cardinality $s$-t vertex cuts of $G$.

AboElFotoh and Colbourn [1] show that \#MINIMUM $s$-t VERTEX CUT is \#Pcomplete.

Let $G=(N, E)$ be an undirected graph and $r \notin N$. Let $V=N \cup r$ and define cost function $c_{G}:\left(\begin{array}{c}V \\ 2\end{array}\right) \rightarrow\{0,1\}$ by

$$
c_{G}(v, w)= \begin{cases}0 & \text { if }\{v, w\} \in E, \quad\left(\{v, w\} \in\left(\begin{array}{l}
V \\
2
\end{array}\right)\right) . \\
1 & \text { otherwise }\end{cases}
$$

Let $G=(N, E)$ be an undirected graph and let $s, t \in N$ be distinct vertices of $G$ which are not adjacent. Let $G^{\prime}=\left(N, E^{\prime}\right)$ be the graph defined by $E^{\prime}=E \cup\{\{s, t\}\}$.

Lemma 3.3: Let $C=\widetilde{c_{G}}$ and $C^{\prime}=\widetilde{c_{G^{\prime}}}$. Then, we have for each $X \subseteq N$

$$
C(X)-C^{\prime}(X)= \begin{cases}1 & \text { if }\{s, t\} \subseteq X \text { and } N-X \text { is an } s \text { - } t \text { vertex cut of } G, \\ 0 & \text { otherwise. }\end{cases}
$$

(Proof) If $\{s, t\} \nsubseteq X$, then $G[X]=G^{\prime}[X]$. Suppose that $\{s, t\} \subseteq X$. If $N-X$ is not an $s$ - $t$ vertex cut of $G$, then the number of connected components of $G^{\prime}$ is same as that of $G$. Otherwise, the number of connected components of $G^{\prime}$ is one less than that of $G$. Therefore, the desired equation (3.2) follows from Proposition 2.4.

Lemma 3.4: Let $F_{j}$ be the number of $s$-t vertex cuts of $G$ of size $j$ for $j=0,1, \cdots, n-$ 2. Then, we have

$$
\Phi_{s}(C)-\Phi_{s}\left(C^{\prime}\right)=\sum_{j=0}^{n-2} F_{j} \frac{j !(n-j-1) !}{n !} .
$$

(Proof) Let us consider the function $f: 2^{N} \rightarrow\{0,1\}$ defined by

$$
f(X)= \begin{cases}1 & \text { if }\{s, t\} \subseteq X \text { and } N-X \text { is an } s \text { - } t \text { vertex cut of } G, \\ 0 & \text { otherwise }\end{cases}
$$


for each $X \subseteq N$. The Shapley value $\Phi_{s}(f)$ is evaluated as follows:

$$
\begin{aligned}
n ! \Phi_{s}(f) & =\sum_{s \in X \subseteq N}(|X|-1) !(n-|X|) !(f(X)-f(X-s)) \\
& =\sum_{\substack{\{s, t\} \subseteq X \subseteq N \\
N-X \text { is an } s-t \text { vertex cut of } G}}(|X|-1) !(n-|X|) ! \\
& \sum_{\substack{Y \subseteq N-\{s, t\} \\
Y \text { is an } s-t \text { vertex cut of } G}}^{n-2}(n-|Y|-1) !|Y| ! \\
& =\sum_{j=0}^{n-2} F_{j}(n-j-1) ! j ! .
\end{aligned}
$$

By Lemma 3.3 and the linearity of the Shapley value, we have the desired equation (3.3).

Theorem 3.5: MCSTG-SHAPLEY is \#P-hard even if the cost functions are restricted to be $\{0,1\}$-valued.

(Proof) We reduce \#MINIMUM $s$ - $t$ VERTEX CUT to MCSTG-SHAPLEY, where we shall use the proof technique used in [3].

Let $G=(N, E)$ be an undirected graph with $s, t \in N$ being non-adjacent. Let $\hat{v}_{1}, \cdots, \hat{v}_{n-2}$ be new vertices disjoint from $N$ and for $i=0,1, \cdots, n-2$ define $G_{i}=$ $\left(N_{i}, E_{i}\right)$ by

$$
\begin{aligned}
& N_{i}=N \cup\left\{\hat{v}_{1}, \cdots, \hat{v}_{i}\right\} \\
& E_{i}=E \cup \bigcup_{j=1}^{i}\left\{\left\{s, \hat{v}_{j}\right\},\left\{\hat{v}_{j}, t\right\}\right\} .
\end{aligned}
$$

Let $F_{j}^{(i)}$ be the number of $s$ - $t$ vertex cuts of $G_{i}$ of size $j$ for $i=0,1, \cdots, n-2$ and $j=0,1, \cdots, n-2+i$. Since the mapping $X \mapsto X \cup\left\{\hat{v}_{1}, \cdots, \hat{v}_{i}\right\}$ is a one-to-one correspondence between the set of $s$ - $t$ vertex cuts of $G$ and that of $G_{i}$, we have

$$
F_{j}^{(i)}= \begin{cases}0 & \text { if } 0 \leq j<i \\ F_{j-i} & \text { if } i \leq j \leq n-2+i\end{cases}
$$

Let $C_{i}=\widetilde{{c_{G_{i}}}}$ and $C_{i}^{\prime}=\widetilde{{c_{G_{i}^{\prime}}}^{\prime}}$ for $i=0,1, \cdots, n-2$. Then, we have from (3.11) and Lemma 3.4 that

$$
\begin{aligned}
(n+i) !\left(\Phi_{s}\left(C_{i}\right)-\Phi_{s}\left(C_{i}^{\prime}\right)\right) & =\sum_{j=i}^{n-2+i} F_{j}^{(i)} j !(n+i-j-1) ! \\
& =\sum_{j=0}^{n-2} F_{j}(i+j) !(n-j-1) !
\end{aligned}
$$

for $i=0,1, \cdots, n-2$. 
Let us denote $(n-j-1) ! F_{j}$ by $f_{j}(j=0,1, \cdots, n-2)$. Now, we have the following system of linear equations:

$$
\sum_{j=0}^{n-2}(i+j) ! f_{j}=(n+i) !\left(\Phi_{s}\left(C_{i}\right)-\Phi_{s}\left(C_{i}^{\prime}\right)\right) \quad(i=0,1, \cdots, n-2) .
$$

Since the determinant of the coefficient matrix of the left-hand side of (3.14) is $\left(\Pi_{j=0}^{n-2} j !\right)^{2}$ (see [4]), the system (3.14) has the unique solution.

Therefore, if we have a polynomial time algorithm for computing the Shapley values $\Phi\left(C_{i}\right)$ and $\Phi\left(C_{i}^{\prime}\right)$ for $i=0,1, \cdots, n-2$, we can compute the right-hand side of system (3.14) in polynomial time. Since the size of each coefficient of the system (3.14) of linear equations are polynomially bounded by $n$, we can compute $f_{j}(j=0,1, \cdots, n-2)$, and hence, $F_{j}(j=0,1, \cdots, n-2)$ by the Gaussian elimination in time polynomial in $n$. In particular, we can compute the number of minimum $s$ - $t$ vertex cuts of $G$ in polynomial time.

\section{Polynomial Cases}

In this section, we consider subclasses of MCST games whose Shapley value can be computed in polynomial time. We begin with MCST games $(N, \tilde{c})$ where the cost functions $c$ are $\{0,1\}$-valued.

A clique $Q \subseteq V$ of a graph $G=(V, E)$ is called a clique cut of $G$ if $Q$ is also a vertex cut of $G$. For a network $\left(\mathrm{K}_{V}, c\right)$, where $c$ is $\{0,1\}$-valued, we define $c^{+}:\left(\begin{array}{c}V \\ 2\end{array}\right) \rightarrow\{0,1\}$ by

$$
c^{+}(v, w)=\left\{\begin{array}{ll}
1 & \text { if } r \in\{v, w\}, \\
c(v, w) & \text { otherwise }
\end{array} \quad\left(\{v, w\} \in\left(\begin{array}{l}
V \\
2
\end{array}\right)\right) .\right.
$$

Lemma 4.1: Suppose that $\left(\mathrm{K}_{V}, c\right)$ is a network with source $r \in V$, where $c$ is $\{0,1\}$ valued and graph $G(0)=(V, E(0))$ is defined by (2.24). Let $Q$ be a clique cut of $G(0)=(V, E(0))$ and $U, W \subseteq V$ are such that $r \in U, U \cup W=V, U \cap W=Q$, $U-Q \neq \emptyset, W-Q \neq \emptyset$ and there exists no edge connecting a vertex in $U-Q$ and $a$ vertex in $W-Q$. Then, we have the followings.

(i) If $r \notin Q$, then

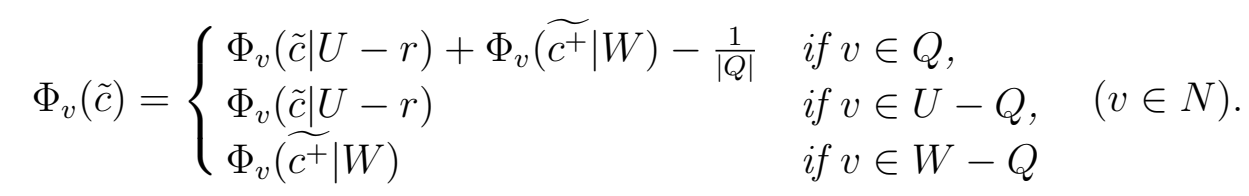

(ii) If $r \in Q$, then

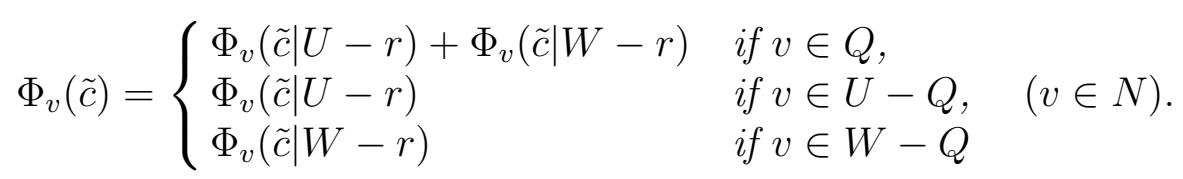


(Proof) We consider (i) only since (ii) can be treated similarly. Let $G=G(0)$ for the sake of notational simplicity. Let $X \subseteq N$ and denote by $\mathcal{C}, \mathcal{C}_{U}$ and $\mathcal{C}_{W}$ the sets of connected components of $G[X \cup r], G[(X \cup r) \cap U]=G[(X \cap U) \cup r]$ and $G[(X \cup r) \cap W]=G[X \cap W]$, respectively.

Suppose $X \cap Q=\emptyset$. Then, $\mathcal{C}$ is the disjoint union of $\mathcal{C}_{U}$ and $\mathcal{C}_{W}$. It follows from Proposition 2.4 that

$$
\tilde{c}(X)=\left|\mathcal{C}_{U}\right|+\left|\mathcal{C}_{W}\right|-1=\tilde{c}(X \cap(U-r))+\widetilde{c^{+}}(X \cap W) .
$$

Suppose $X \cap Q \neq \emptyset$. Since $Q$ is a clique, for each of $\mathcal{C}, \mathcal{C}_{U}$ and $\mathcal{C}_{W}$, there exists a unique component intersecting $Q$. Let us denote these components by $C, C_{U}$ and $C_{W}$, respectively. Then, we have

$$
\mathcal{C}=\left(\mathcal{C}_{U}-C_{U}\right) \cup\left(\mathcal{C}_{W}-C_{W}\right) \cup\{C\}
$$

and it follows from Proposition 2.4 that

$$
\tilde{c}(X)=\left|\mathcal{C}_{U}\right|-1+\left|\mathcal{C}_{W}\right|-1=\tilde{c}(X \cap(U-r))+\widetilde{c^{+}}(X \cap W)-1 .
$$

Summarizing, we have

$$
\tilde{c}(X)=\tilde{c}(X \cap(U-r))+\widetilde{c^{+}}(X \cap W)-f_{Q}(X) \quad(X \subseteq N),
$$

where $f_{Q}: 2^{N} \rightarrow \mathbb{R}$ is defined by

$$
f_{Q}(X)=\left\{\begin{array}{ll}
1 & \text { if } X \cap Q \neq \emptyset, \\
0 & \text { otherwise }
\end{array} \quad(X \subseteq N) .\right.
$$

Therefore, we have from the linearity of $\Phi$ that

$$
\Phi(\tilde{c})=\Phi(\tilde{c} *(U-r))+\Phi\left(\widetilde{c^{+}} * W\right)-\Phi\left(f_{Q}\right) .
$$

Since we have

$$
\Phi_{v}\left(f_{Q}\right)=\left\{\begin{array}{cl}
\frac{1}{|Q|} & \text { if } v \in Q, \\
0 & \text { otherwise }
\end{array} \quad(v \in N)\right.
$$

the desired equation (4.2) follows from (4.9) and Lemma 2.1.

It follows from Lemma 4.1 that for game $(N, \tilde{c})$ where $c$ is $\{0,1\}$-valued, the computation of the Shapley value is reduced to the computation of the Shapley values of games restricted to the 2-connected components of $G(0)$.

A graph $G$ is chordal if $G$ does not contain an induced cycle of length four or more. A vertex $v$ of a graph $G$ is called a simplicial vertex if the neighbors of $v$ induce a clique. A simplicial order of a graph $G=(V, E)$ is an ordering $v_{1}, \cdots, v_{n}$ of vertices of $G$ such that $v_{i}$ is a simplicial vertex of $G\left[\left\{v_{1}, \cdots, v_{i}\right\}\right]$ for $i=1, \cdots, n$, where $n=|V|$.

Theorem 4.2: Suppose that $\left(\mathrm{K}_{V}, c\right)$ is a network with source $r \in V$, where $c$ is $\{0,1\}$-valued and graph $G(0)=(V, E(0))$ is defined by (2.24). If $G(0)$ is chordal, then the Shapley value of game $(N, \tilde{c})$ can be computed in $\mathrm{O}\left(n^{2}\right)$ time. 
(Proof) We let $G=G(0)$. Suppose that $G$ is chordal. Then, $G$ has a simplicial order $v_{1}, \cdots, v_{n+1}$, which can be found in $\mathrm{O}\left(n^{2}\right)$ time using the lexicographical breadth-first search [20]. For $i=1, \cdots, n+1$, let $W_{i}=\left\{v_{1}, \cdots, v_{i}\right\}$ and $Q_{i}$ the set of neighbors of $v_{i}$ in $W_{i}$.

We can inductively compute the Shapley value of $(N, \tilde{c})$ by repeated applications of Lemma 4.1 as follows. Let $i^{*} \in\{1, \cdots, n+1\}$ such that $v_{i^{*}}=r$. We consider only the case when $i^{*} \neq 1$ since the other case is treated similarly. For $i=1, \cdots, i^{*}-1$, since the neighbors $Q_{i}$ of $v_{i}$ is a clique cut of $G\left[W_{i}\right]$, we have from Proposition 2.5 and Lemma 4.1 that

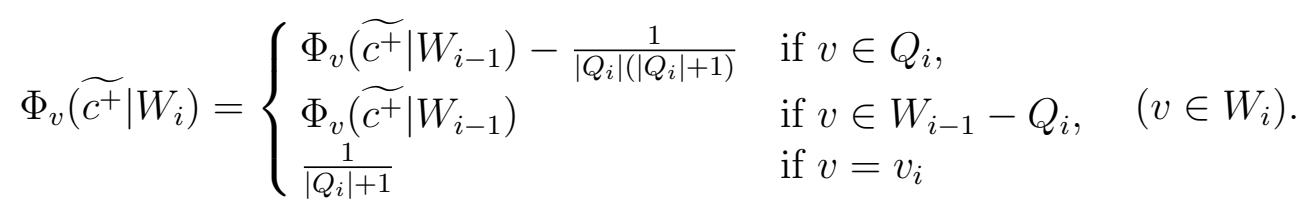

For $i=i^{*}$, we have similarly

$$
\Phi_{v}\left(\widetilde{c} \mid W_{i}-r\right)=\left\{\begin{array}{ll}
\Phi_{v}\left(\widetilde{c^{+}} \mid W_{i-1}\right)-\frac{1}{\left|Q_{i}\right|} & \text { if } v \in Q_{i}, \\
\Phi_{v}\left(\widetilde{c^{+}} \mid W_{i-1}\right) & \text { if } v \in W_{i-1}-Q_{i}
\end{array} \quad\left(v \in W_{i}-r\right) .\right.
$$

For $i=i^{*}+1, \cdots, n+1$, if $r \notin Q_{i}$, then we have

$$
\Phi_{v}\left(\widetilde{c} \mid W_{i}-r\right)= \begin{cases}\Phi_{v}\left(\widetilde{c} \mid W_{i-1}-r\right)-\frac{1}{\left|Q_{i}\right|\left(\left|Q_{i}\right|+1\right)} & \text { if } v \in Q_{i}, \\ \Phi_{v}\left(\widetilde{c} \mid W_{i-1}-r\right) & \text { if } v \in W_{i-1}-Q_{i}, \quad\left(v \in W_{i}-r\right) . \\ \frac{1}{\left|Q_{i}\right|+1} & \text { if } v=v_{i}\end{cases}
$$

Otherwise, we have

$$
\Phi_{v}\left(\widetilde{c} \mid W_{i}-r\right)=\left\{\begin{array}{ll}
\Phi_{v}\left(\widetilde{c} \mid W_{i-1}-r\right) & \text { if } v \in W_{i-1}, \quad\left(v \in W_{i}-r\right) . \\
0 & \text { if } v=v_{i}
\end{array} \quad .\right.
$$

It is now obvious the overall computation of the Shapley value takes $\mathrm{O}\left(n^{2}\right)$ time.

Next, we consider MCST games $(N, \tilde{c})$, where $c$ is not necessarily $\{0,1\}$-valued. For a network $\left(\mathrm{K}_{V}, c\right)$, where $c:\left(\begin{array}{c}V \\ 2\end{array}\right) \rightarrow \mathbb{R}_{+}$is arbitrary, and $\alpha \in \mathbb{R}_{+}$, we define $G(\alpha)=(V, E(\alpha))$ by

$$
E(\alpha)=\left\{\{v, w\} \mid\{v, w\} \in\left(\begin{array}{c}
V \\
2
\end{array}\right), c(v, w) \leq \alpha\right\}
$$

A function $c:\left(\begin{array}{c}V \\ 2\end{array}\right) \rightarrow \mathbb{R}_{+}$is called an ultrametric if for each distinct $x, y, z \in V$ we have

$$
c(x, z) \leq \max \{c(x, y), c(y, z)\} .
$$

Equivalently, $c$ is an ultrametric if and only if for each distinct $x, y, z \in V$ the maximum of $c(x, y), c(y, z), c(z, x)$ is attained by at least two pairs.

Proposition 4.3: Let $\left(\mathrm{K}_{V}, c\right)$ be a network. Then, $c$ is an ultrametric if and only if for each $\alpha \in \mathbb{R}_{+}$all the connected components of $G(\alpha)$ are complete. 
(Proof) Suppose that $c:\left(\begin{array}{c}V \\ 2\end{array}\right) \rightarrow \mathbb{R}_{+}$is an ultrametric. Let $\alpha \geq 0$ and let us consider two arbitrary vertices $v, w$ in a component of $G(\alpha)$. Then, there exists a path

$$
v=v_{0}, v_{1}, \cdots, v_{l}=w
$$

in $G(\alpha)$ from $v$ to $w$. By definition of $G(\alpha)$, we have

$$
c\left(v_{i-1}, v_{i}\right) \leq \alpha \quad(i=1, \cdots, l) .
$$

We show that for $i=1, \cdots, l$

$$
c\left(v_{0}, v_{i}\right) \leq \alpha .
$$

For $i=1$ this is trivial. Let $k \geq 2$ and suppose that for $i=k-1$ (4.19) holds. Then, since $c$ is an ultrametric, we have by the induction hypothesis that

$$
c\left(v_{0}, v_{k}\right) \leq \max \left\{c\left(v_{0}, v_{k-1}\right), c\left(v_{k-1}, v_{k}\right)\right\} \leq \alpha .
$$

Therefore, $\{v, w\}$ is an edge of $G(\alpha)$.

Conversely, suppose that $c$ is not an ultrametric. Then, there exist distinct $x, y, z \in V$ such that $c(x, z)>\max \{c(x, y), c(y, z)\}$. For $\alpha=\max \{c(x, y), c(y, z)\}$, $x$ and $z$ are in the same connected component of $G(\alpha)$ but $\{x, z\}$ is not an edge of $G(0)$.

If $c:\left(\begin{array}{c}V \\ 2\end{array}\right) \rightarrow \mathbb{R}_{+}$is an ultrametric, the number of distinct values of $c(v, w)$ is at most $|V|-1=n$ (see [21]). Hence, it follows from Propositions 2.3, 4.3 and 2.5 that $\Phi(\tilde{c})$ can be computed in $\mathrm{O}\left(n^{3}\right)$ time. However, it is possible to have an $\mathrm{O}\left(n^{2}\right)$ time algorithm for computing $\Phi(\tilde{c})$ (see [5] and [2]).

A connected subgraph of a tree is called a subtree. A function $c:\left(\begin{array}{c}V \\ 2\end{array}\right) \rightarrow \mathbb{R}_{+}$is called a subtree distance [16] if there exist a tree $T=(X, F)$, a function $l: F \rightarrow \mathbb{R}_{+}$ and a family $\left(T_{v} \mid v \in V\right)$ of subtrees of $T$ indexed by $V$ such that

$$
c(v, w)=d_{T}\left(T_{v}, T_{w}\right) \quad\left(\{v, w\} \in\left(\begin{array}{c}
V \\
2
\end{array}\right)\right),
$$

where $d_{T}\left(T_{v}, T_{w}\right)$ is the minimum length of a path connecting a vertex of $T_{v}$ and a vertex of $T_{w}$ with respect to the length function $l$.

Lemma 4.4: Let $\left(\mathrm{K}_{V}, c\right)$ be a network. If $c$ is a subtree distance, then $G(\alpha)$ is chordal for each $\alpha \in \mathbb{R}_{+}$.

(Proof) We call the pair $(T, l)$ of an undirected tree $T=(X, F)$ and a function $l: F \rightarrow$ $\mathbb{R}_{+}$a weighted tree. Let $d$ be a positive integer. For a weighted tree $(T=(X, F), l)$, where $l(x, y)>0$ for all $\{x, y\} \in F$, we call $|T| \subseteq \mathbb{R}^{d}$ an embedding of $(T, l)$ if there exists an injection $\psi: X \rightarrow \mathbb{R}^{d}$ such that

(i) $|T|=\bigcup_{\{x, y\} \in F}[\psi(x), \psi(y)]$,

(ii) $\|\psi(x), \psi(y)\|_{2}=l(x, y) \quad(\{x, y\} \in F)$,

(iii) $\left[\psi\left(x_{1}\right), \psi\left(y_{1}\right)\right] \cap\left[\psi\left(x_{2}\right), \psi\left(y_{2}\right)\right] \neq \emptyset$ implies $\left\{x_{1}, y_{1}\right\} \cap\left\{x_{2}, y_{2}\right\} \neq \emptyset$

$$
\left(\left\{x_{1}, y_{1}\right\},\left\{x_{2}, y_{2}\right\} \in F\right),
$$


where $[p, q] \subseteq \mathbb{R}^{d}$ denotes the line segment with end-points $p, q \in \mathbb{R}^{d}$. For an embedding $|T|$ of $(T, l)$, a closed connected subset of $|T|$ is called a subtree of $|T|$. For a subtree $R$ of $|T|$ and $\alpha \geq 0$, define

$$
R^{+\alpha}=\left\{p|p \in| T \mid, d_{|T|}(p, R) \leq \alpha\right\},
$$

where

$$
d_{|T|}(p, R)=\min \left\{d_{|T|}(p, q) \mid q \in R\right\}
$$

and $d_{|T|}(p, q)$ is the length of the unique path connecting $p$ and $q$ in $|T|$. Then, $R^{+\alpha}$ is again a subtree of $|T|$.

Let $c:\left(\begin{array}{c}V \\ 2\end{array}\right) \rightarrow \mathbb{R}_{+}$be a subtree distance. Then, there exist a weighted tree $(T=$ $(X, F), l)$ and a family $\left(T_{v}=\left(X_{v}, F_{v}\right) \mid v \in V\right)$ of subtrees of $T$ such that (4.21) holds. We can assume without loss of generality that $l(x, y)>0$ for all $\{x, y\} \in F$. Let $|T|$ be an embedding of $(T, l)$ with an injection $\phi: X \rightarrow \mathbb{R}^{d}$ for some $d$. For each $v \in V$, the embedding $|T|$ of $T$ naturally induces embedding of $T_{v}$ :

$$
\left|T_{v}\right|=\bigcup_{\{x, y\} \in F_{v}}[\psi(x), \psi(y)]
$$

which is a subtree of $|T|$.

For $\alpha \geq 0$, let us consider the family $\left(\left|T_{v}\right|^{+\frac{\alpha}{2}} \mid v \in V\right)$ of subtrees of $|T|$. Then, $\left|T_{v}\right|^{+\frac{\alpha}{2}} \cap\left|T_{w}\right|^{+\frac{\alpha}{2}} \neq \emptyset$ if and only if $d_{T}\left(T_{v}, T_{w}\right) \leq \alpha$. Therefore, in the graph $G(\alpha)=$ $(V, E(\alpha))$ defined by (4.15), we have $\{v, w\} \in E(\alpha)$ if and only if $\left|T_{v}\right|^{+\frac{\alpha}{2}}$ and $\left|T_{w}\right|^{+\frac{\alpha}{2}}$ intersect. It follows from [12, Theorem 3] that $G(0)$ is a chordal graph.

By Proposition 2.2, Lemma 4.4 and Theorem 4.2, we have the following theorem.

Theorem 4.5: Let $\left(\mathrm{K}_{V}, c\right)$ be a network. If $c$ is a subtree distance, then the Shapley value of game $(N, \tilde{c})$ can be computed in $\mathrm{O}\left(n^{4}\right)$ time.

\section{Summary and Concluding Remarks}

We showed that computing the Shapley value of MCST games is \#P-hard even if the cost functions are restricted to be $\{0,1\}$-valued. We also investigated MCST games whose Shapley values can be computed in polynomial time. We showed that if the cost function of the given networks is a subtree distance, then the Shapley value can be computed in $\mathrm{O}\left(n^{4}\right)$ time, where $n$ is the number of players.

For future research, it would be interesting to investigate the computational complexity of approximation of the Shapley value of MCST games. Also, finding a class of MCST games for which the Shapley value can be computed efficiently, which possibly extends the class given in this paper, would be an interesting research topic as well.

\section{Acknowledgments}

The author is grateful to Hiroshi Hirai for valuable suggestions and to Shuji Kijima, Satoru Iwata and Satoru Fujishige for helpful comments and discussions. 


\section{References}

[1] H. M. AboElFotoh and C. J. Colbourn: Computing 2-terminal reliability for radio-broadcast networks. IEEE Transactions on Reliability 38 (1989) 538-555.

[2] K. Ando and S. Kato: Reduction of ultrametric minimum cost spanning tree games to cost allocation games on rooted trees. RIMS Preprint No. 1674, Research Institute for Mathematical Sciences, Kyoto University, June 2009 (to appear in Journal of the Operations Research Society of Japan).

[3] H. Aziz, O. Lachish, M. Paterson and R. Savani: Power indices in spanning tree games. In: A. Goldberg and Y. Zhou (eds.): Proceedings of the 5th International Conference on Algorithmic Aspects in Information and Management - AAIM 2009 (Lecture Notes in Computer Science 5564) (Springer-Verlag, 2009), pp. 5567.

[4] R. Bacher: Determinants of matrices related to the Pascal triangle. Journal de Théorie des Nombres de Bordeaux 14 (2002) 19-41.

[5] G. Bergantiños and J. J. Vidal-Puga: The optimistic TU game in minimum cost spanning tree problems. International Journal of Game Theory 36 (2007) 223-239.

[6] C. G. Bird: On cost allocation for a spanning tree. A game theoretic approach. Networks 6 (1976) 335-350.

[7] A. Bogomolnaia and H. Moulin: Sharing a minimal cost spanning tree: Beyond the Folk solution. Games and Economic Behavior (to appear).

[8] B. Dutta and A. Kar: Cost monotonicity, consistency and minimum cost spanning tree games. Games and Economic Behavior 48 (2004) 223-248.

[9] U. Faigle, W. Kern, S. P. Fekete and W. Hochstättler: On the complexity of testing membership in the core of min-cost spanning tree games. International Journal of Game Theory 26 (1997) 361-366.

[10] U. Faigle, W. Kern and J. Kuipers: Computing the nucleolus of min-cost spanning tree games is NP-hard. International Journal of Game Theory 27 (1998) 443-450.

[11] V. Feltkamp, S. Tijs and S. Muto: On the irreducible core and the equal remaining obligations rule of minimum cost spanning extension problems. CentER Discussion Paper No.94106, Center for Economic Research, Tilburg University, 1994.

[12] F. Gavril: The intersection graphs of subtrees in trees are exactly the chordal graphs. Journal of Combinatorial Theory Series B 16 (1974) 47-56.

[13] D. Granot and G. Huberman: Minimum cost spanning tree games. Mathematical Programming 21 (1981) 1-18. 
[14] D. Granot and G. Huberman: The relationship between convex games and minimum cost spanning tree games: a case for permutationally convex games. SIAM Journal on Algebraic and Discrete Methods 3 (1982) 288-292.

[15] D. Granot and G. Huberman: On the core and nucleolus of minimum cost spanning tree games. Mathematical Programming 29 (1984) 323-347.

[16] H. Hirai: Characterization of the distance between subtrees of a tree by the associated tight span. Annals of Combinatorics 10 (2006) 111-128.

[17] A. Kar: Axiomatization of the Shapley value on minimum cost spanning tree games. Games and Economic Behavior 38 (2002) 265-277.

[18] J. B. Kruskal: On the shortest spanning subtree of a graph and the traveling salesman problem. Proceedings of the American Mathematical Society 7 (1956) $48-50$.

[19] H. Norde, S. Moretti and S. Tijs: Minimum cost spanning tree games and population monotonic allocation schemes. European Journal of Operational Research 154 (2004) 84-97.

[20] D. J. Rose, R. E. Tarjan and G. S. Lueker: Algorithmic aspect of vertex elimination on graphs. SIAM Journal on Computing 5 (1976) 266-283.

[21] C. Semple and M. Steel: Phylogenetics (Oxford University Press, 2003).

[22] L. S. Shapley: A value for n-person games. In: H. W. Kuhn and A. W. Tucker (eds.): Contributions to the Theory of Games, volume II (Princeton University Press, 1953), pp. 307-317.

[23] E. Winter: The Shapley value. In: R. J. Aumann and S. Hart (eds.): Handbook of Game Theory, Volume 3 (Elsevier, 2002). 